\title{
Comparing the effects of implicit and explicit temporal expectation on choice response time and response conflict
}

\author{
Melisa Menceloglu $^{1}$ (D) Marcia Grabowecky ${ }^{1,2} \cdot$ Satoru Suzuki $^{1,2}$
}

Published online: 28 October 2016

(C) The Psychonomic Society, Inc. 2016

\begin{abstract}
People can use temporally structured sensory information to anticipate future events. Temporal information can be presented implicitly through probability manipulation without participants' awareness of the manipulation, or explicitly conveyed through instructions. We examined how implicit and explicit temporal information established temporal expectations that influenced choice response times and response conflict (measured as flanker effects). We implicitly manipulated temporal structure by block-wise varying the likely timing of a target. In the short-interval block, a target was presented frequently ( $80 \%$ of trials) after a short $(400 \mathrm{~ms})$ cue-to-target interval and infrequently ( $20 \%$ of trials) after a long $(1200 \mathrm{~ms})$ interval; the probability assignment was reversed in the long-interval block. Building on this baseline condition (Experiment 1), we augmented the temporal information by filling the cue-to-target intervals with tones (Experiment 2), explicitly informed participants of the prevalent time interval (Experiment 3) and provided trial-by-trial reminders of the prevalent time interval (Experiment 4). The temporal probability manipulation alone (of which participants were unaware) influenced choice response times but only when the temporal information was augmented with tones, whereas providing the explicit knowledge of the temporal manipulation, with or without trial-by-trial reminders, robustly influenced choice response times. Response conflict was unaffected by these conditions. These results suggest that
\end{abstract}

Melisa Menceloglu

mencel@u.northwestern.edu

1 Department of Psychology, Northwestern University, 2029 Sheridan Rd., Evanston, IL 60208, USA

2 Interdepartmental Neuroscience Program, Northwestern University, Evanston, IL, USA temporal expectation can be established by the implicit learning of a temporal structure given that sufficiently strong temporal information is presented as well as by the explicit knowledge of the temporal structure. This established temporal expectation influences choice response times without necessarily affecting the strength of response conflict.

Keywords Temporal processing $\cdot$ Visual perception

\section{Introduction}

Just as predictions about where something will happen can improve task performance (Luck, Hillyard, Mouloua, \& Hawkins, 1996; Posner, 1980; for a review, see Funes, Lupiáñez, \& Milliken, 2005), predictions about when something will happen can be beneficial in many tasks. In fact, we constantly use temporally structured sensory or motor information to build expectations about when a future event of interest is likely or unlikely to occur, and these temporal expectations influence our reaction to the event (for reviews, see Coull \& Nobre, 2008; Nobre, Correa, \& Coull, 2007). A simple case would be experiencing rhythmically repeating events that generate an expectation that events will continue to occur on the beat. It has been shown that whether people are aware or unaware of the rhythmicity of the preceding stimulus sequence, they tend to respond faster to an event that occurs on the anticipated beat (Bolger, Coull, \& Schön, 2014; Praamstra, Kourtis, Kwok \& Oostenveld, 2006; Praamstra, Kourtis, Kwok \& Oostenveld, 2006; Rohenkohl, Coull, \& Nobre, 2011), suggesting that periodic rhythms automatically establish temporal expectations.

While rhythmic stimuli are somewhat special, temporal expectation can be more generally established from the statistically learning of the likely timing of occurrence of task- 
relevant events. This has been demonstrated using the foreperiod paradigm (Näätänen, 1970; Niemi \& Näätänen, 1981) in which the time interval between a temporal cue and a target event, termed the foreperiod, is probabilistically manipulated. When the foreperiod is constant within a block of trials, the target onset is highly predictable, resulting in strong temporal expectation. In contrast, when the foreperiod is varied within a block of trials, the target onset becomes less predictable, resulting in weaker temporal expectation. Studies using the foreperiod paradigm have shown that responses to targets become faster as temporal expectation becomes stronger (Los, Knol, \& Boers, 2001; Mattes \& Ulrich, 1997). These results suggest that temporal expectation can be established based on the statistical learning of the prevalent foreperiod. However, it is unclear whether this learning is implicit. That is, it is unclear whether people would automatically learn the prevalent foreperiod and anticipate responding at the expected timing without being aware of any statistical variation in the foreperiods. Rhythm-based temporal expectation can be established through the implicit encoding of rhythmicity, as discussed above. However, it does not necessarily follow that temporal expectation based on learning of the prevalent foreperiod could be established implicitly because the encoding of a rhythm and the learning of the statistically prevalent foreperiod may depend on interdependent but different mechanisms (Teki, Grube. Griffiths, 2012; Teki, Grube, Kumar, Griffiths, 2011).

In contrast to the uncertainty regarding whether temporal expectation can be established through the implicit learning of the statistically prevalent foreperiod, it has been shown that the explicit knowledge of the likely foreperiod can reliably establish temporal expectation. For example, Coull and Nobre (1998) provided a cue on each trial to indicate the likely time interval between the cue and a target, similar to an endogenous spatial-cueing paradigm in which a cue conveys the likely spatial location of a target (Posner, 1980). As in spatialcueing studies, temporal cueing studies have shown that the processing of stimuli appearing at a cued time is enhanced (relative to those appearing at a different time) as indicated by faster responses and increased neural activation in sensory and motor cortices (Correa, Lupiáñez, Milliken, \& Tudela, 2004; Correa, Lupiáñez, \& Tudela, 2006; Lange, Krämer, \& Röder, 2006).

Several studies compared the effects of the statistical learning and the explicit knowledge of the prevalent time interval on temporal expectation using the phenomenon known as the attentional blink - a deficit in identifying the second target (T2) when it closely follows the first target (T1) within a rapid stream of distractors (Raymond, Shapiro, \& Arnell, 1992). In general, when the timing of $\mathrm{T} 2$ relative to $\mathrm{T} 1$ was predictable, attentional blink was attenuated (Martens \& Johnson, 2005; Shen \& Alain 2011; 2012; Visser, Ohan, \& Enns, 2015; Visser, Tang, Badcock, \& Enns, 2014). In particular,
Martens and Johnson (2005) compared the effects of the learning and the explicit knowledge of the likely T1-T2 interval on the attenuation of attentional blink. For measuring the effects of learning, Martens and Johnson (2005) compared T2 detection deficits between a constant T1-T2 interval condition (always $270 \mathrm{~ms}$ or always $720 \mathrm{~ms}$ within each block of trials), in which the constant T1-T2 interval could be learned, and a variable T1-T2 interval condition $(270 \mathrm{~ms}$ and $720 \mathrm{~ms}$ intermixed). There was no performance difference between the two conditions, suggesting that the use of constant intervals did not enable the learning of the critical duration to establish temporal expectation. For measuring the effects of explicit knowledge, they always varied the T1-T2 intervals, but in one condition they provided a cue on each trial informing participants about the T1-T2 interval on the upcoming trial $(270 \mathrm{~ms}$ or $720 \mathrm{~ms})$. T2 detection deficits were significantly attenuated by the informative cues, suggesting that explicit knowledge of timing established temporal expectation. The authors argued that this dissociation between the lack of temporal expectation from statistical learning and effective temporal expectation from explicit temporal knowledge might stem from participants' inability to extract temporal information solely based on statistical structures and/or their inability to maintain temporal information in working memory. Using a similar paradigm, Visser et al. (2014) showed that $\mathrm{T} 2$ detection deficits were attenuated in a constant T1-T2 interval condition relative to a variable T1-T2 interval condition given that participants were informed of the prevalent timing ( $270 \mathrm{~ms}$ or $720 \mathrm{~ms})$ at the beginning of each block of trials. This result suggests that people are able to keep the temporal information active in working memory but are unable to extract it from experience.

Other studies, however, have demonstrated that the temporal information that reduces $\mathrm{T} 2$ detection deficits can be learned from experience under appropriate conditions (Shen \& Alain, 2012; Visser et al., 2015). Shen and Alain (2012) used an auditory version of the attentional blink task and block-wise manipulated the relative probability $(80 \%$ or $20 \%$ ) of a short T2 lag (lagging 2 items from T1) and a long T2 lag (lagging 8 items from T1). Although they did not inform participants of the probability manipulation, T2 detection deficits for the short $\mathrm{T} 2 \mathrm{lag}$ were reduced when the short lag was prevalent ( $80 \%$ ) compared with when it was rare (20\%), suggesting that temporal expectation could develop from the statistical learning of the prevalent temporal interval. Because much research has demonstrated that the auditory modality tends to dominate the visual modality in temporal processing (Aschersleben \& Bertelson, 2003; Chen \& Yeh, 2009; Lukas, Philipp, \& Koch, 2014; Ortega, GuzmanMartinez, Grabowecky, \& Suzuki, 2014), this result may suggest that stronger processing of temporal information facilitates the learning of temporal structure underlying temporal expectation. 
Nevertheless, Visser et al. (2015) suggested that even when temporal information is visually presented, the establishment of temporal expectation through statistical learning can be demonstrated using a sensitive measure of $\mathrm{T} 2$ detection deficits. By manipulating the relative probabilities of different T1T2 lags, they generated two temporal structures, one in which the conditional probability of $\mathrm{T} 2$ increased with longer lagsthe "aging" condition - and the other in which the probability of T2 was greatest at the shortest lag - the "non-aging" condition (Näätänen, 1970). If temporal expectations were to be established from learning these temporal structures, for the longest lag, T2 detection deficits should be reduced to a greater degree in the aging compared with the non-aging condition, whereas for the shortest lag T2 detection, deficits should be reduced to a greater degree in the non-aging compared with the aging condition. When Visser et al. (2015) used accuracy to measure $\mathrm{T} 2$ detection deficits, they demonstrated only the superiority of the aging condition for the longest lag. However, when they made the task speeded and used response times as a more sensitive measure of $\mathrm{T} 2$ detection deficits (and also increased the sample size), they demonstrated both the superiority of the aging condition for the longest lag and the superiority of the non-aging condition for the shortest lag. These results suggest that relatively subtle effects of temporal expectation from the learning of temporal structure can be demonstrated using a speeded response time task.

Taken together, previous research has demonstrated that temporal expectation from the explicit knowledge of a prevalent time interval reliably facilitates stimulus identification at an expected time, whereas temporal expectation developed through the experience of likely and unlikely time intervals produces mixed effects. Although the results from Shen and Alain (2012) and Visser et al. (2015) suggested that temporal expectation can be established from the experience of temporal structure without explicit instructions about the temporal structure, it is unclear whether participants in those experiments were aware or unaware of the temporal structures that they learned. The goal of the current study was to investigate systematically how the strength and the awareness of temporal information influence the establishment of temporal expectation.

In Experiment 1, we elicited temporal expectation by block-wise manipulating the relative probability of short and long cue-to-target intervals (e.g. Menceloglu, Grabowecky, \& Suzuki, 2016). In the short-interval block, a target stimulus was frequently ( $80 \%$ of the trials) presented following a short $(400 \mathrm{~ms})$ post-cue interval and infrequently ( $20 \%$ of the trials) following a long $(1,200 \mathrm{~ms})$ post-cue interval. In the longinterval block, a target stimulus was frequently ( $80 \%$ of the trials) presented following a long $(1,200 \mathrm{~ms})$ post-cue interval and infrequently (20\% of the trials) following a short (400 ms) post-cue interval. This consistent temporal structure may be sufficient to elicit temporal expectation, anticipating a target stimulus $400 \mathrm{~ms}$ after the cue in the short-interval block and 1,200 $\mathrm{ms}$ after the cue in the long-interval block. We measured the strength of temporal expectation as the speeding of response times on the short-interval trials in the shortinterval block, when a target was presented at the expected time - the expected-timing condition - relative to the shortinterval trials in the long-interval block, when a target was presented at an unexpected time - the unexpected-timing condition. Greater speeding indicated stronger temporal expectation. Note that long-interval trials were uninformative, because if the target did not appear after the short interval, it necessarily had to appear after the long interval. Because participants could reorient their attention to the long interval upon not receiving the target after the short interval even in the short-interval block, the expected-timing versus unexpectedtiming distinction would be degenerate on long-interval trials (Correa et al., 2004; Coull \& Nobre, 1998).

In Experiment 2, we tested whether increasing the strength of temporal information might strengthen the implicit learning of temporal information. As we discussed, much evidence suggests that the auditory modality provides stronger temporal information than the visual modality under normal circumstances (Chen \& Yeh, 2009; Lukas, Philipp, \& Koch, 2014; Ortega, Guzman-Martinez, Grabowecky, \& Suzuki, 2014). We thus reasoned that filling the cue-to-target interval with auditory tones may increase the strength of temporal information (Steinborn \& Langner, 2011). This manipulation was also motivated by the fact that a manipulation of temporal structure facilitated T2 detection in an auditory (Shen and Alain, 2012) but not visual (Martens \& Johnson, 2005) attentional blink paradigm (see above). Importantly, to determine whether temporal expectation can be established from the implicit learning of temporal information, we conducted a post-experiment interview to confirm that participants were unaware of the temporal structure.

In Experiment 3, we tested whether explicit knowledge of the temporal manipulation might strengthen temporal expectation, by informing participants of the prevalent cue-to-target interval at the beginning of each block of trials. In Experiment 4, we increased the salience of the explicit knowledge by reminding participants of the likely cue-to-target interval on every trial. Such trial-by-trial reminding is often used in studies investigating the effects of temporal expectation (Correa et al., 2004; Correa et al., 2006). Comparison between Experiments 3 and 4 will inform the degree to which temporal expectation may be strengthened by reminding participants of the likely cue-to-target interval on every trial over and above generally informing participants of the temporal manipulation.

In all experiments, we used a choice response-time task with a mandatory $1,650-\mathrm{ms}$ response window to measure the effects of temporal expectation with high sensitivity because Visser et al. (2015) showed that a temporal expectation effect that was undetected with an accuracy measure was detected 
with a response time measure with a speeded task (see above). In addition, we presented each target stimulus with congruent or incongruent flankers (Eriksen \& Eriksen, 1974) to create two levels of difficulty to guard against potential ceiling or floor effects. A second reason for the inclusion of flankers was that Correa, Cappucci, Nobre, and Lupiáñez (2010) reported that temporal expectation increased flanker effects involving response conflict (i.e., when the to-be-ignored flankers and the central target are assigned different responses). By using congruent and incongruent flankers, we were able to determine how implicitly and explicitly elicited temporal expectation influenced visual selection involving response conflict.

In summary, we systematically investigated how the strength of temporal expectation depended on the strength of implicit temporal information and the salience of explicit temporal information: (1) by only block-wise manipulating the relative probability of the long and short cue-to-target intervals (providing minimal temporal information; Experiment 1); (2) by filling the cue-to-target intervals with tones (increasing the strength of the temporal information; Experiment 2); (3) by informing participants of the block-wise probability manipulation (explicitly providing the temporal information; Experiment 3); and (4) by reminding participants of the likely cue-to-target interval on every trial (increasing the salience of the explicit temporal information; Experiment 4). We used a sensitive speeded response time task in combination with a flanker paradigm that allowed us to avoid ceiling/floor effects and to investigate how temporal expectation elicited by implicit and explicit temporal information influenced visual selection involving response conflicts.

\section{Experiment 1: Temporal manipulation only}

\section{Methods \\ Participants}

Eighteen Northwestern University undergraduate students (11 females) participated. They were between ages 18 and 22 years $(M=19.11, S D=1.13)$. In this and all other experiments, participants had normal or corrected-to-normal vision and normal hearing. They participated for partial course credit, gave informed consent, and were treated according to the guidelines of the Institutional Review Board at Northwestern University.

\section{Stimuli and procedure}

Stimuli were presented using a 13-inch, 2.4-GHz MacBook Pro, running MATLAB (Version R2013b) with Psychtoolbox extensions (Version 3.10.11; Brainard, 1997; Kleiner, Brainard, \& Pelli, 2007; Pelli, 1997). All visual stimuli were black $\left(1.2 \mathrm{~cd} / \mathrm{m}^{2}\right)$ on a light gray background $\left(73 \mathrm{~cd} / \mathrm{m}^{2}\right)$ presented at the center of the display monitor.

Each trial began with the cue display containing a central row of three asterisks $\left(2.6^{\circ} \times 0.7^{\circ}\right.$ visual angle $)$ presented within a black, rectangular frame $\left(22.6^{\circ} \times 17^{\circ}\right.$ visual angle $)$ (Fig. 1, top row). The cue was displayed for $400 \mathrm{~ms}$ and was then replaced by a central fixation dot $\left(0.5^{\circ}\right.$ visual angle diameter) that lasted either $400 \mathrm{~ms}$ - the short interval-or $1,200 \mathrm{~ms}$ - the long interval (Fig. 1, second row). In the short-interval block, the short interval was used $80 \%$ of the time and the long interval was used $20 \%$ of the time. In the long-interval block, the probabilities were reversed. Following the short or the long interval, a target letter replaced the fixation dot. The central target letter was either " $\mathrm{B}$ " or " $\mathrm{D}$ " $\left(0.7^{\circ}\right.$ by $1^{\circ}$ visual angle), and participants were instructed to press the 'g' key (marked with "B") upon detecting the B target and the "h' key (marked with "D") upon detecting the $\mathrm{D}$ target as quickly and as accurately as possible, using the index and middle fingers of their dominant hands. The central target letter was flanked on the left and right sides by either a $\mathrm{B}$ or a D (Fig. 1, third row; $0.25^{\circ}$ visual angle inter-letter spacing, center-to-center). Thus, "BBB" and "DDD" constituted the congruent trials and "DBD" and "BDB" constituted the incongruent trials. The target display lasted $350 \mathrm{~ms}$, followed

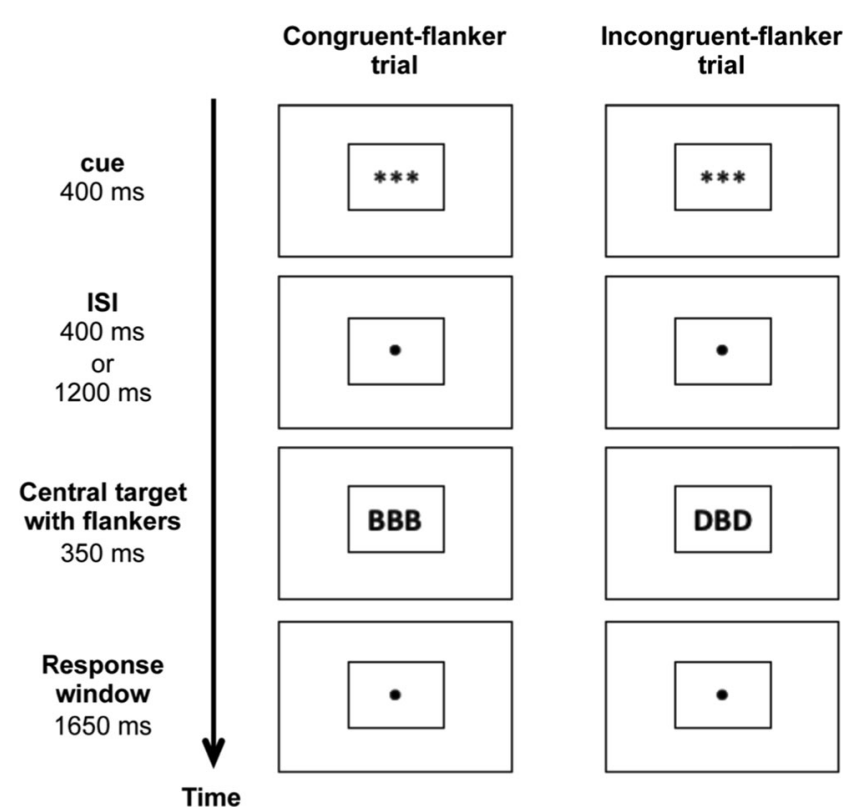

Fig. 1 Illustration of the event sequences for a congruent-flanker trial (left column) and an incongruent-flanker trial (right column). The interstimulusinterval (ISI) was predominantly short (400 $\mathrm{ms}$ on $80 \%$ of the trials and $1,200 \mathrm{~ms}$ on $20 \%$ of the trials) or predominantly long $(1,200 \mathrm{~ms}$ on $80 \%$ of the trials and $400 \mathrm{~ms}$ on $20 \%$ of the trials) within a block of trials. The (interstimulus interval) ISI period was filled with an auditory tone in Experiment 2. Participants indicated whether the central target letter was "B" or "D" by pressing the corresponding response key. The target letter was always flanked either by the same target letters (congruent-flanker trials) or by the other target letters (incongruent-flanker trials). Participants were required to respond within $1650 \mathrm{~ms}$ after the target 
by the central fixation display (Fig. 1, bottom row). Participants were required to respond within the $1,650 \mathrm{~ms}$ response window; otherwise the trial was classified as incorrect. The next trial began following a 1,500 ms intertrial interval during which a blank screen was shown.

The asterisk cue and the letters were in Calibri font. The viewing distance was $65 \mathrm{~cm}$, and participants were instructed to maintain central fixation throughout the experiment. Although auditory stimuli were presented only in Experiment 2, we had participants wear headphones in all experiments to promote contextual similarity across the experiments.

The experiment was divided into two blocks, a shortinterval block and a long-interval block, with a total of 380 trials. The order of the blocks was counterbalanced across participants. There were four trial types with Temporal Expectation (expected-timing [80\%] or unexpected-timing [20\%]) and Target-Flanker Congruity (congruent [50\%] or incongruent [50\%]) as the two factors. Within a block, trials were presented in a random order. Short breaks were given half way through each block and between blocks. The first ten trials in each block served as practice trials and were excluded from analysis; therefore, there were 180 experimental trials in each block.

\section{Data acquisition and analysis}

In this and all other experiments, response time (RT) and accuracy (proportion correct) data were recorded for each trial. In the reported RT analyses, we used RTs from all correctresponse trials without trimming or transformation. However, we also repeated the entire RT analyses after removing RT outliers (outside of three standard deviations from the mean in each condition) from each participant; none of the statistical results changed, suggesting that the reported results are not driven by RT outliers. A two-way repeated measures analysis of variance (ANOVA) was performed on RT and accuracy data from the short-interval trials (see the Introduction section for rationale) with Temporal Expectation (expected-timing vs. unexpected-timing) and Target-Flanker Congruity (congruent vs. incongruent) as the within-subject factors.

\section{Results and Discussion}

Overall response accuracy was high $(M=0.97 ; S E=0.007)$. There was no main effect of Temporal Expectation, TargetFlanker Congruity, or their interaction on accuracy $(p s>0.2)$.

For RTs, the main effect of Temporal Expectation was not significant; participants responded equally fast on the expected-timing $(M=703 ; S E=36.85)$ and unexpectedtiming $(M=694 ; S E=32.73)$ trials, $F(1,17)=0.61, p=$ 0.45 . The main effect of Target-Flanker Congruity was significant; participants responded faster on the congruent trials $(M=678 ; S E=34.31)$ than on the incongruent trials $(M=$ $720 ; S E=34.66), F(1,17)=46.97, p<0.001, \eta_{\mathrm{p}}{ }^{2}=0.73$. The interaction was not significant, $F(1,17)=0.18, p=0.67$ (Fig. 2). Thus, the probability manipulation alone did not produce a temporal expectation effect despite the fact that we obtained a robust congruity effect.

\section{Experiment 2: Temporal manipulation + tone-filled intervals}

\section{Methods}

\section{Participants}

Twenty Northwestern University undergraduate students were recruited to participate. Data from two participants were excluded from the analyses; one participant failed to follow instructions correctly, and the other participant did not pay attention to the task. The final sample included 18 participants (10 females) between ages 18 and 20 years $(M=18.5, S D=$ $0.71)$.

\section{Stimuli and procedure}

The stimuli and procedure were the same as in Experiment 1 except for the following. We used a 15 -inch, 2.4-GHz MacBook Pro. Crucially, each cue-to-target interval was filled with a $4,410-\mathrm{Hz}$ tone ( $60 \mathrm{~dB}$ SPLA) binaurally presented over headphones. Participants were told that the tones were task irrelevant.

\section{Results and Discussion}

Overall response accuracy was high $(M=0.93 ; S E=0.017)$. There was a significant main effect of Target-Flanker Congruity; participants were more accurate on the congruent trials $(M=0.95 ; S E=0.014)$ than on the incongruent trials $(M$ $=0.91 ; S E=.023), F(1,17)=4.73, p=0.04, \eta_{\mathrm{p}}{ }^{2}=0.22$. Neither the main effect of Temporal Expectation nor its interaction with Target-Flanker Congruity was significant ( $p s>$ 0.2 ). The accuracy results suggested no speed-accuracy trade-off as the RT result showed the same trend (see below).

For RTs, the main effect of Temporal Expectation was significant; participants responded faster on the expected-timing trials $(M=590 ; S E=18.21)$ than on the unexpected-timing trials $(M=614 ; S E=17.19), F(1,17)=6.73, p=0.02, \eta_{\mathrm{p}}{ }^{2}=$ 0.28 . The main effect of Target-Flanker Congruity also was significant; participants responded faster on the congruent trials $(M=586 ; S E=19.39)$ than on the incongruent trials $(M=$ 617; $S E=15.43), F(1,17)=16.43, p=0.001, \eta_{\mathrm{p}}{ }^{2}=0.49$. The 


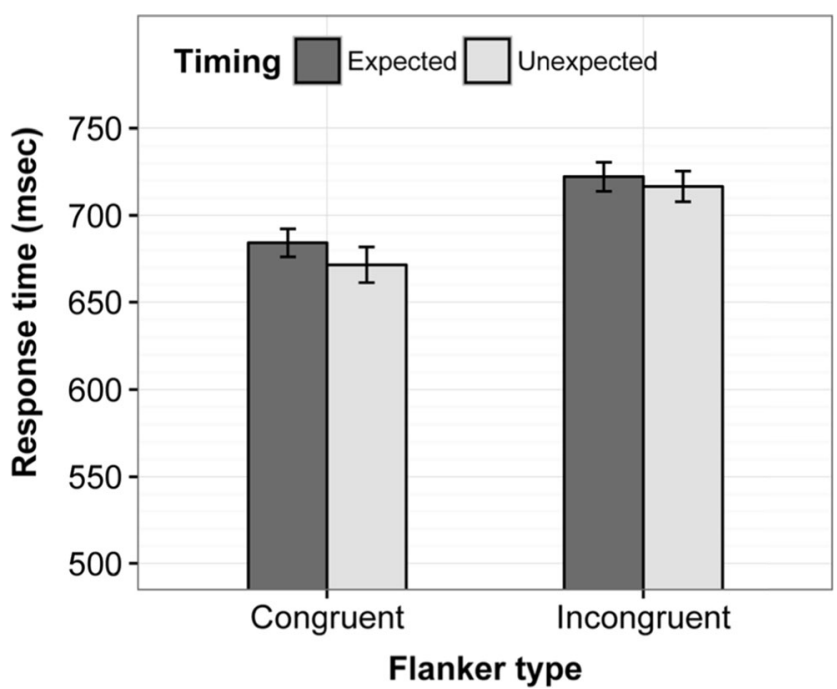

Fig. 2 Results from Experiment 1 with temporal manipulation only. Mean response times for classifying the central letter accompanied by the congruent (left bars) or incongruent (right bars) flankers, presented with the expected (dark gray bars) or unexpected (light gray bars) timing. Error bars represent \pm 1 standard error of the mean adjusted for withinparticipants comparisons (Morey, 2008)

interaction was not significant, $F(1,17)=0.10, p=0.75$ (Fig. 3).

During post-experiment debriefing, 13 of the 18 participants expressed no knowledge of any variations in temporal intervals between blocks or within a block. Two participants remembered that there were short and long tones, but did not notice anything about their relative prevalence. Two participants noted that the tones were longer in one block than in the other block. One participant recognized the probability

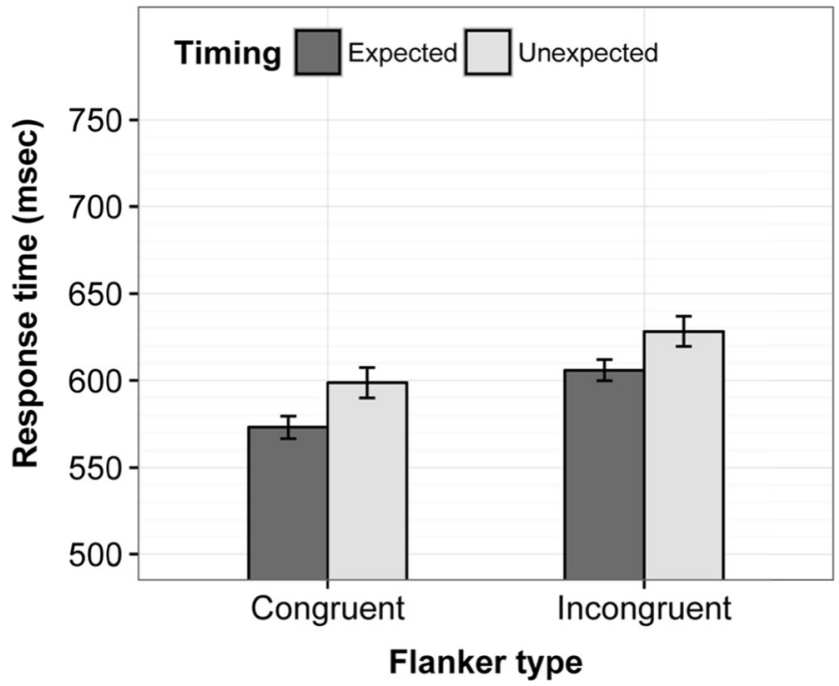

Fig. 3 Results from Experiment 2 with tone-filled cue-to-target intervals. Mean response times for classifying the central letter accompanied by the congruent (left bars) or incongruent (right bars) flanker letters, presented with the expected (dark gray bars) or unexpected (light gray bars) timing. Error bars represent \pm 1 standard error of the mean adjusted for withinparticipants comparisons (Morey, 2008) manipulation. Importantly, the main effect of Temporal Expectation remained significant without the three participants who were potentially aware of the probability manipulation, $F(1,14)=7.11, p=0.018, \eta_{\mathrm{p}}{ }^{2}=0.34$. Thus, increasing the strength of temporal information by filling the cue-totarget intervals with tones facilitated the implicit learning of the temporal structure that established temporal expectation.

To roughly assess the time course of the implicit statistical learning, we conducted the same analysis but separately for each one-third ${ }^{1}$ of the trials; in other words, we evaluated the temporal expectation effects across three 60-trial time-bins, with each time-bin containing $M=44.7(S D=4.8)$ expected-timing trials and $M=11.1(S D=2.8)$ unexpectedtiming trials with correct responses. Temporal Expectation did not significantly interact with Time-bin, $F(2,34)=0.53, p>$ 0.59 , suggesting that any implicit learning likely developed within less than 60 trials.

\section{Experiment 3: Temporal manipulation + explicit instructions}

\section{Methods}

\section{Participants}

Twenty Northwestern University undergraduate students were recruited. Two participants' data were excluded from the analyses. One of them was overtly inattentive and with accuracy more than 2 SD below the mean; the other one was very lethargic with accuracy more than $3 \mathrm{SD}$ below the mean. Their accuracy rates were detected as outliers based on IQR analysis. The final sample consisted of 18 participants (8 females) between ages 18 and 21 years $(M=18.33, S D=0.77)$.

\section{Stimuli and procedure}

The stimuli and procedure were identical to those in Experiment 1, except that participants were explicitly informed of the timing manipulation and instructed to use this knowledge to improve performance.

\section{Results and Discussion}

Overall response accuracy was high $(M=0.97 ; S E=0.01)$. The main effect of Target-Flanker Congruity was marginal; participants were somewhat more accurate on the congruent trials $(M=0.98, S E=0.005)$ than on the incongruent trials $(M$

\footnotetext{
${ }^{1}$ Had we used a finer time division, we would not have been able to guarantee that each time bin contained all trial types (expected, unexpected, congruent, and incongruent) for all participants.
} 
$=0.97, S E=0.008), F(1,17)=4.20, p=0.056, \eta_{\mathrm{p}}{ }^{2}=0.2$. Neither the main effect of Temporal Expectation nor its interaction with Target-Flanker Congruity was significant ( $p \mathrm{~s}>$ 0.3 ). The accuracy results suggested no speed-accuracy trade-off as the RT result showed the same trend (see below).

For RTs, the main effect of Temporal Expectation was significant; participants responded faster on the expected-timing trials $(M=648 ; S E=25.34)$ than on the unexpected-timing trials $(M=710 ; S E=25.54), F(1,17)=36.75, p<.001, \eta_{\mathrm{p}}{ }^{2}=$ 0.68 . The main effect of Target-Flanker Congruity was also significant; participants responded faster on the congruent trials $(M=657 ; S E=25.03)$ than on the incongruent trials $(M=$ $701 ; S E=25.31), F(1,17)=40.56, p<0.001, \eta_{\mathrm{p}}{ }^{2}=0.71$. The interaction was not significant, $F(1,17)=1.37, p=0.26$ (Fig. 4). Thus, explicit knowledge of the probability manipulation facilitated the establishment of temporal expectation.

\section{Experiment 4: Temporal manipulation + explicit instructions + trial-by-trial reminders}

\section{Methods}

\section{Participants}

Nineteen Northwestern University undergraduate students were recruited. One participant's data were excluded from the analyses with accuracy more than 3 SD below the mean and detection as an outlier based on IQR analysis. The final

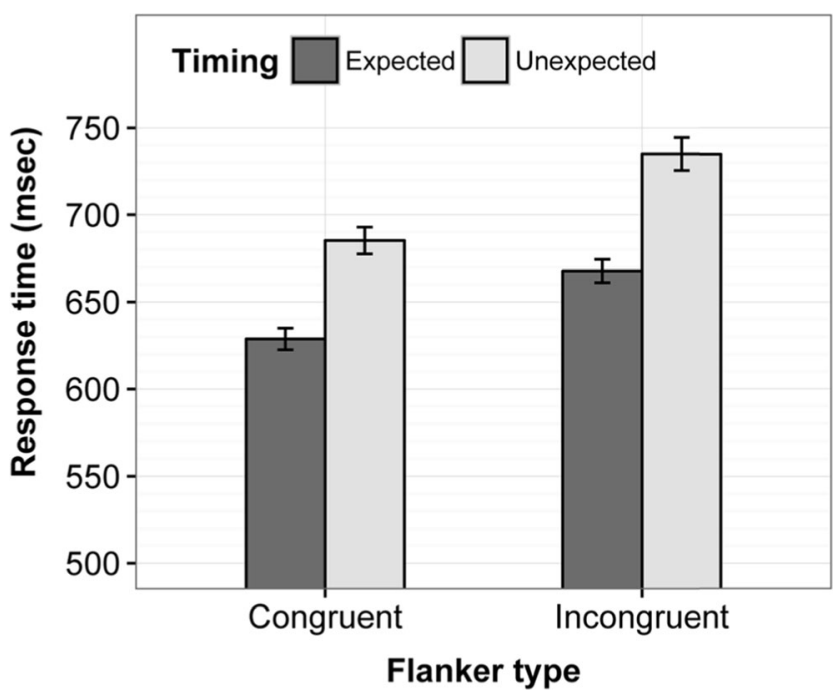

Fig. 4 Results from Experiment 3 with knowledge of temporal manipulation. Mean response times for classifying the central letter accompanied by the congruent (left bars) or incongruent (right bars) flanker letters, presented with the expected (dark gray bars) or unexpected (light gray bars) timing. Error bars represent \pm 1 standard error of the mean adjusted for within-participants comparisons (Morey, 2008) sample consisted of 18 participants ( 9 females) between ages 18 and 20 years $(M=18.33, S D=0.69)$.

\section{Stimuli and procedure}

The stimuli and procedure were the same as in Experiment 3 except that the word "SHORT" or "LONG" (approximately $4^{\circ} \times 1^{\circ}$ visual angle), instead of a row of asterisks, was presented as the cue at the beginning of each trial. In the shortinterval block, the cue was always "SHORT," in the longinterval block the cue was always "LONG," both reminding participants of the frequent cue-to-target interval for the block. Participants were explicitly informed about the timing manipulation and instructed to use the informative trial-by-trial cue to anticipate the timing of the target letter appearance.

\section{Results and Discussion}

Overall response accuracy was high $(M=0.96 ; S E=0.008)$. There was no main effect of Temporal Expectation or TargetFlanker Congruity, or any interaction ( $p \mathrm{~s}>0.1)$.

For RTs, the main effect of Temporal Expectation was significant; participants responded faster on the expected-timing trials $(M=618 ; S E=16.86)$ than on the unexpected-timing trials $(M=679 ; S E=21.33), F(1,17)=26.52, p<0.001, \eta_{\mathrm{p}}{ }^{2}=$ 0.61 . The main effect of Target-Flanker Congruity also was significant; participants responded faster on the congruent trials $(M=627 ; S E=18.32)$ than on the incongruent trials $(M=$ 671; $S E=18.93), F(1,17)=38.05, p<0.001, \eta_{\mathrm{p}}{ }^{2}=0.69$. The interaction was not significant, $F(1,17)=1.46, p=0.24$ (Fig. 5). Thus, unsurprisingly, providing trial-by-trial reminders of the likely cue-to-target intervals in addition to providing explicit knowledge of the probability manipulation produced a robust temporal expectation effect.

\section{Between-experiment comparison}

To compare the strengths of temporal expectation across the four experiments, we examined the Temporal Expectation by Experiment interaction. For accuracy, the interaction was not significant, $F(3,68)=0.21, p=0.89$, as accuracy was near ceiling across all experiments (see above). For RTs, the crucial interaction was significant, $F(3,68)=9.89, p<0.001, \eta_{\mathrm{p}}{ }^{2}=$ 0.30 , indicating that the strength of temporal expectation depended on how temporal information was provided. To examine this dependency, we compared the magnitude of the temporal expectation effect (unexpected RT minus expected RT) across the four experiments (Fig. 6A).

The current study addressed several specific questions. The first question was whether increasing the strength of temporal information by filling the cue-to-target intervals with tones 


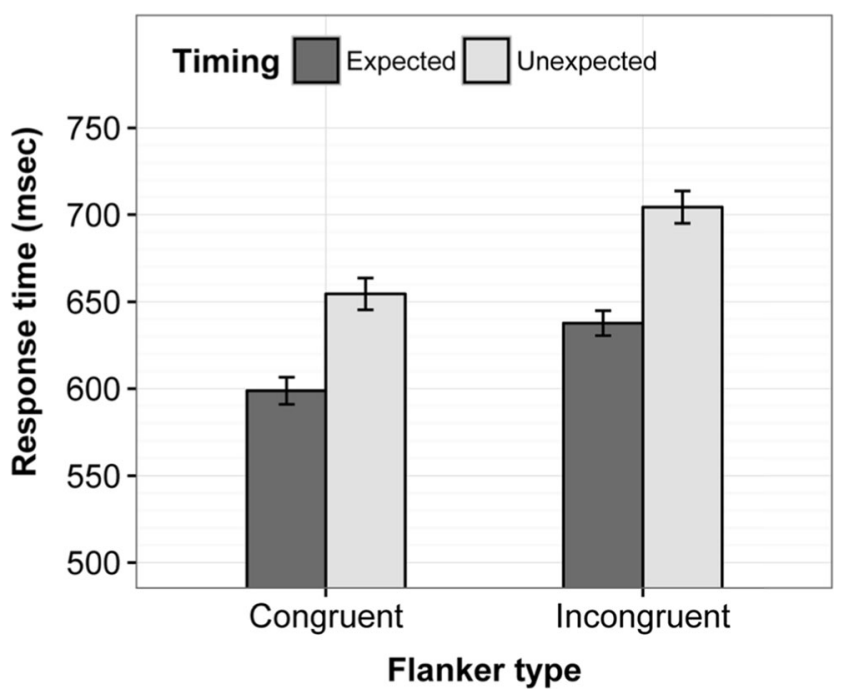

Fig. 5 Results from Experiment 4 with trial-by-trial reminders of temporal manipulation. Mean response times for classifying the central letter accompanied by the congruent (left bars) or incongruent (right bars) flanker letters, presented with the expected (dark gray bars) or unexpected (light gray bars) timing. Error bars represent \pm 1 standard error of the mean adjusted for within-participants comparisons (Morey, 2008)

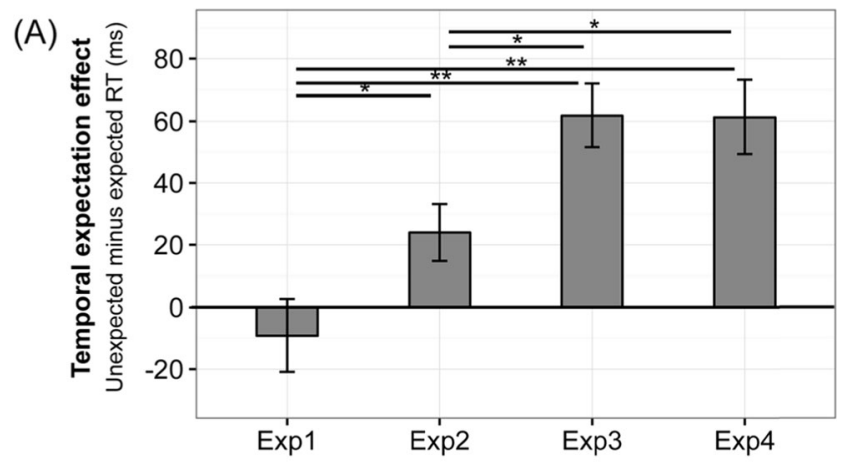

(B)

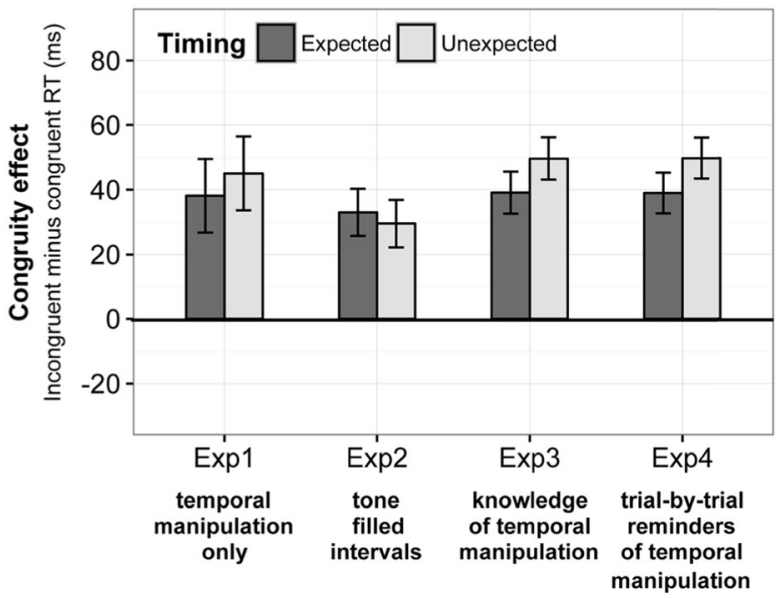

Fig. 6 A Dependence of the temporal expectation effect on the manner in which temporal expectation was elicited in the four experiments. B Dependence of the congruity effect in the expected (dark gray bars) and unexpected (light gray bars) conditions on the manner in which temporal expectation was elicited in the four experiments. Error bars represent \pm 1 standard error of the mean (adjusted for within-participants comparisons for the timing factor in $\mathbf{B}$; Morey, 2008). $* p<0.05$ and $* * p<0.01$ based on pairwise $t$ tests
(Experiment 2) might increase the implicit temporal expectation effect (based on the implicit learning of the temporal structure) relative to having silent (visual only) intervals (Experiment 1). The second question was whether providing explicit temporal information (Experiments 3 and 4) might increase the temporal expectation effect relative to only statistically providing temporal information (Experiments 1 and 2). The third question was whether trial-by-trial reminding of temporal information (Experiment 4) would increase the temporal expectation effect relative to providing only the knowledge of the temporal manipulation (Experiment 3). We addressed these three questions by applying the contrasts $\{-1$, $1,0,0\},\{-1,-1,1,1\}$, and $\{0,0,-1,1\}$, respectively. The first two contrasts were significant, $F(1,68)=4.69, p<0.04, \eta_{\mathrm{p}}{ }^{2}=$ 0.06 and $F(1,68)=24.98, p<0.001, \eta_{\mathrm{p}}{ }^{2}=0.27$, respectively, whereas the third contrast was not, $F(1,68)=0.001, p=0.97$. These results suggest that increasing the strength of temporal information and providing explicit temporal information both increase temporal expectation effects, whereas trial-by-trial reminding of explicit temporal information does not further increase temporal expectation effects (Fig. 6A).

As shown in Fig. 6B, the congruity effect (incongruent RT minus congruent RT) did not depend on the manner of implicitly or explicitly eliciting temporal expectation, nor did the congruity effect differ between the expected-timing (dark gray bars) and unexpected-timing (light gray bars) conditions within each experiment. These results are backed by nonsignificant Congruity by Experiment interaction, $F(3,68)=0.76, p>$ 0.5 , Congruity by Temporal Expectation interaction, $F(1,68)$ $=1.20, p>0.28$, and Congruity by Temporal Expectation by Experiment interaction, $F(3,68)=0.33, p>0.8$.

\section{General Discussion}

Temporal expectation refers to the ability to predict the timing of a future event on the basis of the temporal structure of sensory or motor information, which can facilitate task performance (for reviews, see Coull \& Nobre, 2008; Nobre, Correa, $\&$ Coull, 2007). Prior research showed that, whereas explicit temporal expectation based on knowledge of the prevalent time interval reliably facilitated stimulus identification at the expected time (Correa et al., 2004; Correa et al., 2006; Martens and Johnson, 2005; Visser et al., 2014), implicit temporal expectation elicited through the experience of likely and unlikely time intervals produced mixed results (Martens and Johnson, 2005; Shen \& Alain, 2012; Visser et al., 2015). The goal of the current study was to investigate systematically how the strength of implicit temporal information and the salience of explicit temporal information influenced the effects of temporal expectation on choice response time and visual selection involving response conflicts.

Our attempt to elicit implicit temporal expectation solely by manipulating the relative probability of the long and short 
cue-to-target intervals (e.g., $80 \%$ long and $20 \%$ short implicitly establishing temporal expectation for the long interval) produced little effect on choice response time (Experiment 1). This is consistent with prior studies that produced mixed results using similar probability manipulations. However, filling the cue-to-target intervals with tones generated robust temporal expectation effects despite the fact that participants were still unaware of the probability manipulation (Experiment 2). Because prior research has demonstrated the dominance of the auditory modality over the visual modality in temporal processing (Aschersleben \& Bertelson, 2003; Chen \& Yeh, 2009; Lukas, Philipp, \& Koch, 2014; Ortega, Guzman-Martinez, Grabowecky, \& Suzuki, 2014), this result is consistent with the interpretation that increasing the strength of temporal information by providing redundant temporal information through the auditory modality (for a review, see Shi \& Burr, 2016) may facilitate the establishment of implicit temporal expectation.

Using a visual attentional blink task, Martens and Johnson (2005) reported that temporal expectation effects were absent unless participants were informed of the temporal manipulation of target probability and performance was measured using accuracy. Using an auditory attentional blink task, Shen and Alain (2012) demonstrated temporal expectation effects without informing their participants of the temporal manipulation. Using a visual attentional blink task, but with a sensitive speeded response time measure, Visser et al. (2015) demonstrated temporal expectation effects without informing their participants of the temporal manipulation. These prior results suggest that the strength of temporal information (likely stronger for the auditory than visual modality) and the sensitivity of the behavioral measure (speeded response time likely more sensitive than accuracy) are crucial for demonstrating temporal expectation effects in the absence of informing participants of the temporal manipulation. We used a sensitive speeded response time measure, but did not demonstrate temporal expectation effects in Experiment 1, seeming to contradict Visser et al. (2015). We speculate that temporal information was more strongly presented in Visser et al. (2015) than in the current study because they used an attentional blink paradigm where the passage of time was rhythmically articulated by the rapid and periodic presentations of the distractors following the presentation of $\mathrm{T} 1$; thus, it is possible that the duration of the foreperiod (i.e., the time interval between $\mathrm{T} 1$ and $\mathrm{T} 2$ ) was more strongly encoded in their attentional blink task than in our Experiment 1 where a blank fixation screen was presented during the foreperiod. Consistent with this interpretation, by filling the foreperiod with a tone to provide redundant temporal information through the auditory modality, we were able to demonstrate temporal expectation effects in Experiment 2. Our results extend prior results by demonstrating that temporal expectation can be established based on the implicit learning of temporal structure without awareness of the temporal manipulation in fewer than 60 trials, given that sufficiently strong temporal information is provided.

Consistent with prior results, providing explicit knowledge of a temporal manipulation substantially increased the temporal expectation effect (Experiment 3). Although trial-by-trial reminders of the likely time interval are often provided in addition to the knowledge of temporal probability manipulation (Correa et al., 2004; Correa et al., 2006), comparing our results from Experiments 3 and 4 suggest no additional benefit of providing trial-by-trial reminders. This suggests that people can effectively maintain the knowledge of the prevalent time interval in working memory across many trials.

Interestingly, the flanker congruity effect was unaffected by temporal expectation. The congruity effect was robust and equivalent across the four experiments for both the expected-timing and unexpected-timing trials (Fig. 6B) regardless of the strength of the temporal expectation effect (Fig. 6A). In contrast, Correa et al. (2010) reported that a congruity effect that involved response conflict (like ours) was increased on the expected-timing trials relative to the unexpected-timing trials. This seeming discrepancy suggests an interesting possibility. Given that the temporal manipulations were similar between the two studies (foreperiods of $400 \mathrm{~ms}$ and $1,300 \mathrm{~ms}$ presented with relative probabilities of $75 \%$ and $25 \%$ in Correa et al., 2010, and foreperiods of $400 \mathrm{~ms}$ and 1,200 ms presented with relative probabilities of $80 \%$ and $20 \%$ in the current study), we suspect that the discrepant flanker results likely reflect differences in the stimulus and/or response parameters. Correa et al. (2010) used arrow stimuli that may have produced stronger response conflicts than did our letter stimuli. Stronger baseline flanker effects may be more sensitive for revealing any modulation by temporal expectation. However, the overall magnitude of the flanker effect in our study was no smaller than that in their study. Correa et al. (2010) used stimulus-response mapping that was ideomotor compatible whereas our stimulus-response mapping was arbitrary. Correa et al. (2010) used the " $z$ " and " $m$ " keys that are horizontally separated by a substantial distance on the keyboard to have their participants respond to the left-pointing and right-pointing target arrows, respectively. Thus, their stimulus-response mapping was ideomotor compatible in that a motor action to the left was required to respond to a left-pointing arrow and a motor action to the right was required to respond to a right-pointing arrow (Greenwald \& Shulman, 1973). In our study, the stimulus-response mapping (left and righty keys assigned to the two letter targets) was arbitrary. It is reasonable to hypothesize that ideomotor compatible actions involving fluent response preparations may not be susceptible to response conflicts unless the general state of response preparation to any stimulus (the target or the flankers) are indiscriminately heightened by temporal orientation (Correa et al., 2010). In contrast, actions based on an arbitrary stimulus-response mapping requiring effortful 
response preparation (the current study) may be generally susceptible to response conflict. Furthermore, arrows are a special type of symbolic stimulus that has been shown to direct attention to locations to which they point (Pratt and Hommel, 2003; Ristic and Kingstone, 2006; Ristic, Wright, \& Kingstone, 2007; Reuss, Pohl, Kiesel, \& Kunde, 2011). It is possible that attention-directing effects of arrow stimuli may be enhanced by temporal orientation. If so, the central arrow might have more strongly directed attention to the flanking arrows during temporal orienting, thereby increasing the flanker effects.

\section{Conclusions}

Using a speeded choice response-time task sensitive to temporal expectation (Visser et al., 2015), we investigated how the implicit learning of temporal structure and explicit knowledge of temporal manipulation influenced the strength of temporal expectation. The implicit learning of temporal structure speeded response times only when the strength of temporal information was increased by filling the time intervals with tones, whereas providing the explicit knowledge of temporal manipulation equivalently speeded response times whether participants were simply informed of the temporal manipulation or received trial-by-trial reminders of the likely time interval. Neither implicitly nor explicitly established temporal expectation influenced visual or response selection as measured with a letter flanker task using an arbitrary stimulusto-response mapping.

Acknowledgments This study was supported by a National Institutes of Health grant (R01 EY021184).

\section{References}

Aschersleben, G., \& Bertelson, P. (2003). Temporal ventriloquism: crossmodal interaction on the time dimension 2: Evidence from sensorimotor synchronization. International Journal of Psychophysiology, 50, 157-163.

Bolger, D., Coull, J. T., \& Schön, D. (2014). Metrical rhythm implicitly orients attention in time as indexed by improved target detection and left inferior parietal activation. Journal of Cognitive Neuroscience, 26(3), 593-605.

Brainard, D. H. (1997). The psychophysics toolbox. Spatial Vision, 10, 433-436.

Chen, K., \& Yeh, S. (2009). Asymmetric cross-modal effects in time perception. Acta Psychologica, 130(3), 225-234.

Correa, A., Cappucci, P., Nobre, A. C., \& Lupiáñez, J. (2010). The two sides of temporal orienting: Facilitating perceptual selection, disrupting response selection. Experimental Psychology, 57(2), $142-148$.

Correa, A., Lupiáñez, J., Milliken, B., \& Tudela, P. (2004). Endogenous temporal orienting of attention in detection and discrimination tasks. Perception \& Psychophysics, 66, 264-278.
Correa, A., Lupiáñez, J., \& Tudela, P. (2006). The attentional mechanism of temporal orienting: Determinants and attributes. Experimental Brain Research, 169, 58-68.

Coull, J. T., \& Nobre, A. C. (1998). Where and when to pay attention: The neural systems for directing attention to spatial locations and to time intervals as revealed by both PET and fMRI. The Journal of Neuroscience, 18, 7426-7435.

Coull, J. T., \& Nobre, A. C. (2008). Dissociating explicit timing from temporal expectation with fMRI. Current Opinion in Neurobiology, 18, 137-144.

Eriksen, B. A., \& Eriksen, C. W. (1974). Effects of noise letters upon identification of a target letter in a non- search task. Perception and Psychophysics, 16, 143-149.

Funes, M. J., Lupiáñez, J., \& Milliken, B. (2005). The role of spatial attention and other processes on the magnitude and time course of cueing effects. Cognitive Processing, 6(2), 98-116.

Greenwald, A. G., \& Shulman, H. G. (1973). On doing two things at once: II. Elimination of the psychological refractory period effect. Journal of Experimental Psychology, 101, 70-76.

Kleiner M, Brainard D, \& Pelli D (2007). "What's new in Psychtoolbox3 ?" Perception 36 ECVP Abstract Supplement.

Lange, K., Krämer, U. R., \& Röder, B. (2006). Attending points in time and space. Experimental Brain Research, 173, 130-140.

Los, S. A., Knol, D. L., \& Boers, R. M. (2001). The foreperiod effect revisited: Conditioning as a basis for nonspecific preparation. Acta Psychologica, 106(1), 121-145.

Luck, S. J., Hillyard, S. A., Mouloua, M., \& Hawkins, H. L. (1996). Mechanisms of visual-spatial attention: Resource allocation or uncertainty reduction? Journal of Experimental Psychology: Human Perception \& Performance, 22(3), 725-737.

Lukas, S., Philipp, A. M., \& Koch, I. (2014). Crossmodal attention switching: Auditory dominance in temporal discrimination tasks. Acta Psychologica, 153, 139-146.

Martens, S., \& Johnson, A. (2005). Timing attention: Cuing target onset interval attenuates the attentional blink. Memory \& Cognition, 33(2), 234-240.

Mattes, S., \& Ulrich, R. (1997). Response force is sensitive to the temporal uncertainty of response stimuli. Perception and Psychophysics, 59(7), 1089-1097.

Menceloglu, M., Grabowecky, M., \& Suzuki, S. (2016). Temporal expectation weights visual signals over auditory signals. Psychonomic Bulletin \& Review, in press.

Morey, R. D. (2008). Confidence intervals from normalized data: A correction to Cousineau (2005). Tutorials in Quantitative Methods for Psychology, 4, 61-64.

Näätänen, R. (1970). The diminishing time-uncertainty with the lapse of time after the warning signal in reaction-time experiments with varying foreperiods. Acta Psychologica, 34, 399-418.

Niemi, P., \& Näätänen, R. (1981). Foreperiod and simple reaction time. Psychological Bulletin, 89, 133-162.

Nobre, A. C., Correa, A., \& Coull, J. T. (2007). The hazards of time. Current Opinion in Neurobiology, 17(4), 465-470.

Ortega, L., Guzman-Martinez, E., Grabowecky, M., \& Suzuki, S. (2014). Audition dominates vision in duration perception irrespective of salience, attention, and temporal discriminability. Attention, Perception and Psychophysics, 76, 1485-1502.

Pelli, D. G. (1997). The VideoToolbox software for visual psychophysics: Transforming numbers into movies. Spatial Vision, 10, 437-442.

Posner, M. I. (1980). Orienting of attention. The Quarterly Journal of Experimental Psychology, 32(1), 3-25.

Praamstra, P., Kourtis, D., Kwok, H. F., \& Oostenveld, R. (2006). Neurophysiology of implicit timing in serial choice reaction-time performance. Journal of Neuroscience, 26, 5448-5455.

Pratt, J., \& Hommel, B. (2003). Symbolic control of visual attention: The role of working memory and attentional control settings. Journal of 
Experimental Psychology: Human Perception \& Performance, 29, 835-845.

Raymond, J. E., Shapiro, K. L., \& Arnell, K. M. (1992). Temporary suppression of visual processing in an RSVP task: An attentional blink? Journal of Experimental Psychology: Human Perception and Performance, 18(3), 849-860.

Reuss, H., Pohl, C., Kiesel, A., \& Kunde, W. (2011). Follow the sign! Top-down contingent capture of masked arrow cues. Advances in Cognitive Psychology. 7, 82-91.

Ristic, J., \& Kingstone, A. (2006). Attention to arrows: Pointing to a new direction. Quarterly Journal of Experimental Psychology, 59(11), 1921-1930.

Ristic, J., Wright, A., \& Kingstone, A. (2007). Attentional control and reflexive orienting to gaze and arrow cues. Psychonomic Bulletin and Review, 14(5), 964-969.

Rohenkohl, G., Coull, J. T., \& Nobre, A. C. (2011). Behavioural dissociation between exogenous and endogenous temporal orienting of attention. Plos One, 6, e14620.

Shen, D., \& Alain, C. (2011). Temporal attention facilitates short-term consolidation during a rapid serial auditory presentation task. Experimental Brain Research, 215(3), 285-292.
Shen, D., \& Alain, C. (2012). Implicit temporal expectation attenuates auditory attentional blink. PLoS One, 7(4), e36031.

Shi, Z., \& Burr, D. (2016). Predictive coding of multisensory timing. Current Opinion in Behavioral Sciences, 8, 200-206.

Steinborn, M. B., \& Langner, R. (2011). Distraction by irrelevant sound during foreperiods selectively impairs temporal preparation. Acta Psychologica, 136(3), 405-418.

Teki, S., Grube, M., Kumar, S., \& Griffiths, T. D. (2011). Distinct neural substrates of duration-based and beat-based auditory timing. The Journal of Neuroscience, 31(10), 3805-3812.

Teki, S., Grube, M., \& Griffiths, T. D. (2012). A unified model of time perception accounts for duration-based and beat-based timing mechanisms. Frontiers in Integrative Neuroscience, 5, 90.

Visser, T. A. W., Ohan, J. L., \& Enns, J. T. (2015). Temporal cues derived from statistical patterns can overcome resource limitations in the attentional blink. Attention, Perception, \& Psychophysics, 77(5), $1585-1595$.

Visser, T. A. W., Tang, M. F., Badcock, D. R., \& Enns, J. T. (2014). Temporal cues and the attentional blink: A further examination of the role of expectancy in sequential object perception. Attention, Perception, \& Psychophysics, 76(8), 2212-2220. 